\title{
The Dynamical Behavior of a Rigid Body Relative Equilibrium Position
}

\author{
T.S. Amer \\ Department of Mathematics, Faculty of Science, Tanta University, Tanta 3127, Egypt \\ Correspondence should be addressed to T. S. Amer; tarek.amer@science.tanta.edu.eg
}

Received 26 October 2016; Revised 12 January 2017; Accepted 24 January 2017; Published 8 March 2017

Academic Editor: Manuel De León

Copyright (C) 2017 T. S. Amer. This is an open access article distributed under the Creative Commons Attribution License, which permits unrestricted use, distribution, and reproduction in any medium, provided the original work is properly cited.

In this paper, we will focus on the dynamical behavior of a rigid body suspended on an elastic spring as a pendulum model with three degrees of freedom. It is assumed that the body moves in a rotating vertical plane uniformly with an arbitrary angular velocity. The relative periodic motions of this model are considered. The governing equations of motion are obtained using Lagrange's equations and represent a nonlinear system of second-order differential equations that can be solved in terms of generalized coordinates. The numerical solutions are investigated using the fourth-order Runge-Kutta algorithms through Matlab packages. These solutions are represented graphically in order to describe and discuss the behavior of the body at any instant for different values of the physical parameters of the body. The obtained results have been discussed and compared with some previous published works. Some concluding remarks have been presented at the end of this work. The importance of this work is due to its numerous applications in life such as the vibrations that occur in buildings and structures.

\section{Introduction}

The pendulum models have provided the researchers with a fertile source of examples in nonlinear dynamics and lately in nonlinear control. The most famous rigid pendulum consists of a mass particle that is attached to one end of a massless rigid arm and the other end of the arm is fixed to a pivot point that provides a rotational joint for the arm and mass particle. If the arm and mass particle are constrained to move within a fixed plane, the system is referred to as a planar one-dimension pendulum. If the arm and mass particle are unconstrained, the system is referred to as a spherical twodimension pendulum. The three-dimensional motion of the swinging spring is studied in [1]. The resonance phenomenon that occurs during the motion is also investigated. Nonlinear normal vibration modes of the spring pendulum and the system containing a pendulum absorber are considered in [2].

The published articles on such models are very large. Few researches view the pendulum as a rigid body. Slandered pendulum models are defined by a single rotational degree of freedom, referred to as a planar rigid rotational degree of freedom, or two rotational degrees of freedom, referred to as spherical rigid pendulum. Control problems for planar and spherical pendulum models have been studied by outstanding researchers; see [3-6].

In [7], the motion of a variable length pendulum was studied to determine the characteristics of motion. In [8], the process analysis method is presented as the analytical method to obtain a second-order approximate solution for a simple pendulum. This method does not depend on small parameter and therefore can overcome the disadvantages and limitations of the perturbation expansion method. In [9], the authors studied a simple pendulum with a hinge exhibiting bilinear hysteretic moment-rotation characteristics subjected to periodic base motions. They have shown that the bilinear hysteretic nature of the system becomes an effective way to limit the growth of the response during parametric resonance. Various perturbation techniques [10] were employed to obtain the analytical solutions for many physical problems. In [11], the authors studied the motion of the supported point of a pendulum on an ellipse and the method of small parameter [10] was used to obtain the periodic solution of the equation of motion. The relative periodic motion of a pendulum with an elastic string was studied in [12] and generalized in [13] when the motion of the supported point of a rigid body pendulum with an elastic string moves on an 
elliptical path. The equations of motion were deduced using Lagrange's equations and solved through the small parameter method to obtain their solutions up to the second order of approximation.

In [14] Amer and Bek studied the chaotic responses of a harmonically excited spring pendulum which moves in a circular path under some conditions. The obtained equations of motion represent a nonautonomous system of two nonlinear differential equations of two degrees of freedom. The approximate solution was obtained up to the third order using the multiple scales method [10]. The parametric control of oscillations and rotations of a compound pendulum was studied in [15]. An approximate asymptotic approach of this problem, based on a combination of the averaging method [10] and the maximum principle, is proposed and applied. The limiting cases of small oscillations and rapid rotation of a pendulum are studied in [16].

In [17], the authors studied the vibration and stability of the nonlinear spring pendulum to describe the motion of a ship. The effects of the longitudinal absorber on the system are described through the obtained results. This model is modified in [18] by connecting the spring pendulum to the transverse absorber. So, the motion has three degrees of freedom under multiparametric excitations. The approximate solution is obtained using the multiple scales method up to the second-order approximations.

The nonlinear two-degree-of-freedom system has been examined in [19]. The analytical approximate solution up to the third order is obtained using the same previous method. All the possible resonances of this solution are examined.

The aim of this work is to investigate the motion of a rigid body suspended on an elastic massless spring. The equations of motion are derived using Lagrange's equation and are considered as a nonlinear system of second-order differential equations. Each equation of this system depends on all the body variables with their derivatives. So, it is not easy to separate these equations as explicit second-order differential equations of one variable in one side. In order to overcome this quandary, the Mathematica program is used. Consequently, the numerical solutions are achieved using the fourth-order Runge-Kutta procedure of ode45 solver [20] with the aid of more recent computer package, for example, Matlab program. Computer codes are carried out to obtain the graphical representations of the attained numerical solutions for the different parameters of the body. The stability of the solutions is checked during the time interval of motion. Discussion of the results is presented through the comparison between the different plots for different variables. The importance of this problem is due to its wide applications in many fields such as physics and engineering applications like swaying buildings.

\section{Formulation of the Problem}

This section is devoted to introduce the motion of a rigid body suspended on an elastic massless spring as a pendulum model. So, we consider $O X Y$ as a coordinates system, rotating with angular velocity $\omega$ with respect to the downward axis

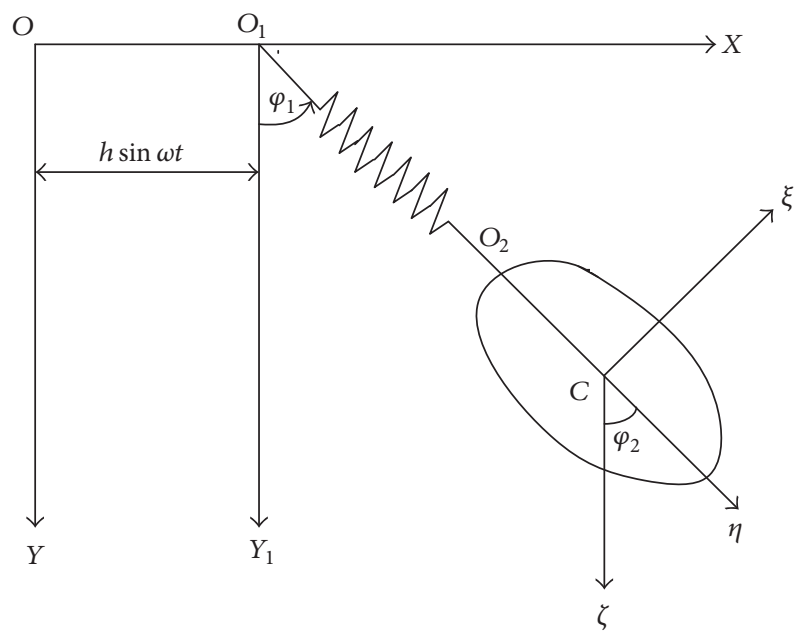

FIgURE 1: The rigid body pendulum.

OY, relative to the motion of a rigid body of mass $m$. The elastic spring is suspended to a point $O_{1}$, where $O O_{1}=$ $h \sin \omega t$ at any time $t$. Let us suppose that the rigid body is attached with the spring at the point $\mathrm{O}_{2}$ and has the point $C$ as a center of mass, $\varphi_{1}$ denotes the deformation angle between the spring and the vertical axis $O_{1} Y_{1}$, and $\varphi_{2}$ refers to the angle between the straight line directed through $\mathrm{O}_{2}$ to $C$ and the vertical. Choosing an orthogonal coordinates system $\mathrm{C} \xi \eta \zeta$ of the body, in which $\mathrm{C \eta}$ directed along $\mathrm{O}_{2} \mathrm{C}, \mathrm{C} \xi$ is perpendicular to $C \eta$ and lying in the $O X Y$ plane, while $C \zeta$ is perpendicular to the $O X Y$ plane (see Figure 1). Assume, without loss of generality, that the axes $C \xi, C \eta$, and $C \zeta$ are the principal axes of inertia of the body.

The coordinates of the center of mass of the body $x_{C}$ and $y_{C}$, relative to the system $O X Y$, can be written as

$$
\begin{aligned}
& x_{C}=h \sin \omega t+\rho \sin \varphi_{1}+a \sin \varphi_{2}, \\
& y_{C}=\rho \cos \varphi_{1}+a \cos \varphi_{2}, \quad a=O_{2} C,
\end{aligned}
$$

where $\rho$ is the length of elastic string after time $t$.

The kinetic energy $T$ and the potential energy $V$ of the system have the form

$$
\begin{aligned}
T & =\frac{1}{2} m\left\{(h \omega \cos \omega t)^{2}+\dot{\rho}^{2}+\left(\rho \dot{\varphi}_{1}\right)^{2}+\left(a \dot{\varphi}_{2}\right)^{2}\right. \\
& +2 h \omega \dot{\rho} \sin \varphi_{1} \cos \omega t+2 h \omega \rho \dot{\varphi}_{1} \cos \omega t \cos \varphi_{1} \\
& +2 a h \omega \dot{\varphi}_{2} \cos \omega t \cos \varphi_{2}+2 a \dot{\rho} \dot{\varphi}_{2} \sin \left(\varphi_{1}-\varphi_{2}\right) \\
& +2 a \dot{\varphi}_{1} \dot{\varphi}_{2} \cos \left(\varphi_{1}-\varphi_{2}\right) \\
& +\omega^{2}\left(h \sin \omega t+\rho \sin \varphi_{1}+a \sin \varphi_{2}\right)^{2}+\frac{J_{3}}{m} \dot{\varphi}_{2}^{2} \\
& \left.+\frac{\omega^{2}}{m}\left(J_{1} \sin ^{2} \varphi_{2}+J_{2} \cos ^{2} \varphi_{2}\right)\right\}, \\
V & =\frac{1}{2} k^{2}(\rho-\ell)^{2}-m g\left(\rho \cos \varphi_{1}+a \cos \varphi_{2}\right),
\end{aligned}
$$

where $J_{1}, J_{2}$, and $J_{3}$ are the principal moments of inertia with respect to the axes $C \xi, C \eta$, and $C \zeta$, respectively, $k^{2}$ is the 
spring's constant, $\ell$ represents the unstretched length of the string, and $g$ denotes the gravitational attraction.

According to the above equations, one can obtain the Lagrangian of the system [21]

$$
L=T-V .
$$

An inspection of (1)-(3), we can observe that the Lagrangian $L$ is expressed in terms of three generalized coordinates $\rho, \varphi_{1}, \varphi_{2}$ and three corresponding generalized velocities $\dot{\rho}, \dot{\varphi}_{1}, \dot{\varphi}_{2}$.

Use the following Lagrange's equations

$$
\begin{aligned}
& \frac{d}{d t}\left(\frac{\partial L}{\partial \dot{q}_{i}}\right)-\frac{\partial L}{\partial q_{i}}=0 ; \\
& q_{i} \equiv\left(\rho, \varphi_{1}, \varphi_{2}\right), \dot{q}_{i} \equiv\left(\dot{\rho}, \dot{\varphi}_{1}, \dot{\varphi}_{2}\right)
\end{aligned}
$$

to obtain the equations of motion in the form

$$
\begin{aligned}
\ddot{\rho}+ & a \ddot{\varphi}_{2} \sin \left(\varphi_{1}-\varphi_{2}\right)-a \dot{\varphi}_{2}^{2} \cos \left(\varphi_{1}-\varphi_{2}\right)-\rho \dot{\varphi}_{1}^{2} \\
& -\omega^{2}\left(2 h \sin \omega t+\rho \sin \varphi_{1}+a \sin \varphi_{2}\right) \sin \varphi_{1} \\
& -g \cos \varphi_{1}+K^{2}(\rho-\ell)=0, \\
\rho \ddot{\varphi}_{1} & +2 \dot{\rho} \dot{\varphi}_{1}+a \ddot{\varphi}_{2} \cos \left(\varphi_{1}-\varphi_{2}\right)+a \dot{\varphi}_{2}^{2} \sin \left(\varphi_{1}-\varphi_{2}\right) \\
& -\omega^{2}\left(2 h \sin \omega t+\rho \sin \varphi_{1}+a \sin \varphi_{2}\right) \cos \varphi_{1} \\
& +g \sin \varphi_{1}=0, \\
\ell_{1} \ddot{\varphi}_{2} & +\left(\ddot{\rho}-\rho \dot{\varphi}_{1}^{2}\right) \sin \left(\varphi_{1}-\varphi_{2}\right) \\
& +\left(\rho \ddot{\varphi}_{1}+2 \dot{\rho} \dot{\varphi}_{1}\right) \cos \left(\varphi_{1}-\varphi_{2}\right) \\
& -\omega^{2}\left(2 h \sin \omega t+\rho \sin \varphi_{1}+a \sin \varphi_{2}\right) \cos \left(\varphi_{1}-\varphi_{2}\right) \\
& +\frac{\omega^{2}}{2 m}\left(J_{2}-J_{1}\right) \sin 2 \varphi_{2}+g \sin \varphi_{2}=0,
\end{aligned}
$$

where

$$
\begin{aligned}
K^{2} & =\frac{k^{2}}{m}, \\
\ell_{1} & =\frac{1}{a}\left(a^{2}-\frac{J_{3}}{m}\right) .
\end{aligned}
$$

Here $\ell_{1}$ is the derived length of the body relative to $O_{2}$. Equations (5) are the governing equations of motion of our model that represent a nonlinear system of second-order differential equations.

In order to study this problem we consider that the oscillations of our system are closing to the position of the relative equilibrium. So, we can assume

$$
J_{1}=J_{2} \text {. }
$$

Hence, for the relative equilibrium state the angles $\varphi_{10}$ and $\varphi_{20}$ are equal, and then we can write

$$
\begin{gathered}
\rho=b+\xi(t), \\
\varphi_{1}=\varphi_{0}+\varphi(t), \\
\varphi_{2}=\varphi_{0}+\psi(t),
\end{gathered}
$$

where $\varphi_{0}$ represents the value of $\varphi_{10}$ and $\varphi_{20}$ and $b$ denotes the pendulum string's length in the case of relative equilibrium. Moreover, the quantities $\varphi_{0}$ and $b$ can be determined from the following equations:

$$
\begin{aligned}
K^{2}(b-\ell) & =\omega^{2}(a+b) \sin ^{2} \varphi_{0}+g \cos \varphi_{0}, \\
g & =\omega^{2}(a+b) \cos \varphi_{0} .
\end{aligned}
$$

Substituting from (8) into (5), then using (7) and (9), we get

$$
\ddot{\xi}+a_{11} \xi+a_{12} \varphi+a_{13} \psi=f_{1},
$$

$$
\begin{aligned}
& b \ddot{\varphi}+a \ddot{\psi}+b_{11} \xi+b_{12} \varphi+b_{13} \psi=f_{2}, \\
& \ell_{1} \ddot{\psi}+b \ddot{\varphi}+c_{11} \xi+c_{12} \varphi+c_{13} \psi=f_{3},
\end{aligned}
$$

where

$$
\begin{aligned}
& a_{11}=K^{2}-\omega^{2}\left(\sin ^{2} \varphi_{0}+2 h \cos \varphi_{0} \sin \omega t\right), \\
& a_{12}=b c_{11} \text {, } \\
& a_{13}=a c_{11}, \\
& b_{11}=c_{11} \text {, } \\
& b_{12}=K^{2}(b-\ell)-\omega^{2} b \cos ^{2} \varphi_{0} \\
& b_{13}=-\omega^{2} a \cos ^{2} \varphi_{0} \text {, } \\
& c_{11}=-\omega^{2} \sin \varphi_{0} \cos \varphi_{0} \\
& c_{12}=-\omega^{2} b \cos ^{2} \varphi_{0} \\
& c_{13}=K^{2}(b-\ell)+\omega^{2}\left(2 h \sin \varphi_{0} \sin \omega t-a \cos ^{2} \varphi_{0}\right) \text {, } \\
& f_{1}=(\xi+b) \dot{\varphi}^{2}+A \xi \varphi+a \ddot{\psi}(\psi-\varphi)+a \dot{\psi}^{2}+B \varphi \psi \\
& +C_{1} \\
& f_{2}=-\xi \ddot{\varphi}-2 \dot{\xi} \dot{\varphi}+(\psi-\varphi) a \dot{\psi}+c_{11}(\xi+b) \varphi^{2} \\
& +\left(D \xi+2 a_{13} \psi\right) \varphi+C_{2}, \\
& f_{3}=\ddot{\xi}(\psi-\varphi)+\left(b \dot{\varphi}^{2}+\xi \dot{\varphi}^{2}\right)(\varphi-\psi)-\xi \ddot{\varphi}-2 \dot{\xi} \dot{\varphi} \\
& +2 \omega^{2} h \sin \omega t \cos \varphi_{0} \\
& -\left[b+a\left(1-\psi^{2}\right)-(b+\xi) \varphi \psi\right] c_{11} \\
& -g \sin \varphi_{0}+\omega^{2} \xi\left(\varphi \cos ^{2} \varphi_{0}-\psi \sin ^{2} \varphi_{0}\right),
\end{aligned}
$$




$$
\begin{aligned}
A= & -2 c_{11}, \\
B= & a \omega^{2} \cos ^{2} \varphi_{0}, \\
D= & \omega^{2} \cos 2 \varphi_{0}, \\
C_{1}= & -b\left(K^{2}-\omega^{2} \sin ^{2} \varphi_{0}\right) \\
& +\omega^{2}\left[2 h \sin \varphi_{0} \sin \omega t+a\left(\sin ^{2} \omega t+\sin ^{2} \varphi_{0}\right)\right] \\
& +g \cos \varphi_{0}+K^{2} \ell, \\
C_{2}= & 2 h \omega^{2} \sin \omega t-(b+a) c_{11}-g \sin \varphi_{0} .
\end{aligned}
$$

Our principle aim is to obtain the numerical solutions of system (10) which consists of three nonlinear differential equations of second-order. In view of the right hand sides of these equations, we found three functions $f_{1}, f_{2}$, and $f_{3}$ given by (12). In fact, it is not easy to obtain the second derivatives of the generalized coordinates $\xi, \varphi$, and $\psi$ such that each equation contains one of these derivatives only.

\section{Numerical Solutions}

This section is devoted to discuss the numerical solutions for the considered model in Section 2. Computer programs are carried out to investigate the graphical representations for these solutions, to describe the motion, and to illustrate the behavior of the pendulum at any time.

System (10) consists of three nonlinear differential equations of second-order in terms of $\xi, \varphi$, and $\psi$ and is reconsidered to obtain the numerical solutions in framework of the fourth-order Runge-Kutta algorithms through Matlab packages [22]. Each equation of this system includes all variables $\xi, \varphi, \psi$ and their derivatives from the first and second order; see systems of (10), (11), (12), and (13). So, the mentioned system is more complicated to deal with and to obtain another corresponding one consisting of secondorder differential equations in terms of $\ddot{\xi}, \ddot{\varphi}$, and $\ddot{\psi}$ explicitly. Computer codes are utilized in order to overcome these difficulties and to separate each of $\ddot{\xi}, \ddot{\varphi}$, and $\ddot{\psi}$. Consequently, system (10) is transformed into the following system with the aid of (11), (12), and (13):

$$
\begin{aligned}
\ddot{\xi}= & -\frac{1}{H}\left\{-a \psi\left\{-(b+\xi)\left(c_{11} \xi+c_{12} \varphi+c_{13} \psi-f_{3}\right)\right.\right. \\
& +b\left(b_{11} \xi+b_{12} \varphi+b_{13} \psi+g \sin \varphi_{0}+\omega^{2}\right. \\
& \cdot\left\{-\xi \varphi \cos ^{2} \varphi_{0}-2 h \sin \omega t \cos \varphi_{0}\right. \\
& +\left[-b+(b+\xi) \varphi \psi+a\left(\psi^{2}-1\right)\right] \\
& \left.\cdot \cos \varphi_{0} \sin \varphi_{0}+\xi \varphi \sin ^{2} \varphi_{0}\right\} \\
& \left.\left.+2 \dot{\xi} \dot{\varphi}-(b+\xi)(\varphi-\psi) \dot{\varphi}^{2}\right)\right\}
\end{aligned}
$$

$$
\begin{aligned}
& +\left[a b-(b+\xi) \ell_{1}\right]\left[C_{1}-a_{11} \xi-a_{12} \varphi\right. \\
& \left.\left.-a_{13} \psi+(b+\xi) \dot{\varphi}^{2}+\left(A \xi+B \psi+a \dot{\psi}^{2}\right) \varphi\right]\right\}, \\
& \ddot{\varphi}=-\frac{1}{b} f_{3}-c_{11} \xi-c_{12} \varphi-c_{13} \psi+\frac{\ell_{1}}{a \psi} \\
& \cdot\left[C_{1}-a_{11} \xi-a_{12} \varphi-a_{13} \psi+(b+\xi) \dot{\varphi}^{2}\right. \\
& \left.+\left(A \xi+B \psi+a \dot{\psi}^{2}\right) \varphi\right] \\
& +\frac{1}{a H \psi}\left\{\ell_{1}\{-a \psi\{(b+\xi)\right. \\
& \cdot\left(f_{3}-c_{11} \xi-c_{12} \varphi-c_{13} \psi\right)+b \\
& \cdot\left(b_{11} \xi+b_{12} \varphi+b_{13} \psi+g \sin \varphi_{0}+\omega^{2}\right. \\
& \cdot\left\{-\xi \varphi \cos ^{2} \varphi_{0}-2 h \sin \omega t \cos \varphi_{0}\right. \\
& +\left[(b+\xi) \varphi \psi+a\left(\psi^{2}-1\right)-b\right] \cos \varphi_{0} \sin \varphi_{0} \\
& \left.\left.\left.+\xi \varphi \sin ^{2} \varphi_{0}\right\}+2 \dot{\xi} \dot{\varphi}-(b+\xi)(\varphi-\psi) \dot{\varphi}^{2}\right)\right\} \\
& +\left[a b-(b+\xi) \ell_{1}\right]\left\{C_{1}-a_{11} \xi-a_{12} \varphi-a_{13} \psi\right. \\
& \left.\left.\left.+(b+\xi) \dot{\varphi}^{2}+\left(A \xi+B \psi+a \dot{\psi}^{2}\right) \varphi\right\}\right\}\right\}, \\
& \ddot{\psi}=-\frac{1}{H}\left\{b(\varphi-\psi)\left[a_{11} \xi+a_{12} \varphi+a_{13} \psi-(A \xi+B \psi) \varphi\right]\right. \\
& -b\left(b_{11} \xi+b_{12} \varphi+b_{13} \psi\right)+(b+\xi)\left(c_{11} \xi+c_{12} \varphi+c_{13} \psi\right) \\
& -b C_{1}(\varphi-\psi)+b \omega^{2} \cos \varphi_{0}\left(\xi \varphi \cos \varphi_{0}+2 h \sin \omega t\right) \\
& -(b+\xi) f_{3}-b g \sin \varphi_{0}+b \omega^{2} \\
& \cdot\{a+b-\psi[(b+\xi) \varphi+a \psi]\} \cos \varphi_{0} \sin \varphi_{0} \\
& \left.-b\left[2 \dot{\xi} \dot{\varphi}+\varphi\left(\omega^{2} \xi \sin ^{2} \varphi_{0}+a(\varphi-\psi) \dot{\psi}^{2}\right)\right]\right\},
\end{aligned}
$$

where

$$
H=\left[a b\left(\psi^{2}-\varphi \psi-1\right)+(b+\xi) \ell_{1}\right] .
$$

It is clear that the left hand sides of the equations of the previous system are given explicitly in terms of $\ddot{\xi}, \ddot{\varphi}$, and $\ddot{\psi}$, respectively. On the other hand, the right hand sides are functions of $\xi, \varphi, \psi, \dot{\xi}, \dot{\varphi}$, and $\dot{\psi}$.

The ode 45 solver is used in order to obtain the numerical solutions of the nonstiff ordinary differential equations of the previous system (14), in which this solver uses a variable step of Runge-Kutta technique [20]. So we can rewrite system (14) as a system of coupled first-order differential equations as follows. 
A choice of the state variables for this system is

$$
\begin{aligned}
& X_{1}=\xi, \\
& X_{2}=\varphi, \\
& X_{3}=\psi, \\
& X_{4}=\dot{\xi}, \\
& X_{5}=\dot{\varphi}, \\
& X_{6}=\dot{\psi},
\end{aligned}
$$

which results in the following state-equations:

$$
\begin{aligned}
& \dot{X}_{1}=X_{4}, \\
& \dot{X}_{2}=X_{5}, \\
& \dot{X}_{3}=X_{6} .
\end{aligned}
$$

Use (16) and (17) into system (14) to get

$$
\begin{aligned}
\dot{X}_{4}= & -\frac{1}{H}\left\{-a X_{3}\left\{-\left(b+X_{1}\right)\right.\right. \\
& \cdot\left(c_{11} X_{1}+c_{12} X_{2}+c_{13} X_{3}-f_{3}\right) \\
& +b\left(b_{11} X_{1}+b_{12} X_{2}+b_{13} X_{3}+g \sin \varphi_{0}+\omega^{2}\right. \\
& \cdot\left\{-X_{1} X_{2} \cos ^{2} \varphi_{0}-2 h \sin \omega t \cos \varphi_{0}\right. \\
& +\left[-b+\left(b+X_{1}\right) X_{2} X_{3}+a\left(\psi^{2}-1\right)\right] \cos \varphi_{0} \sin \varphi_{0} \\
& \left.+X_{1} X_{2} \sin ^{2} \varphi_{0}\right\} \\
& \left.\left.+2 \dot{X}_{1} \dot{X}_{2}-\left(b+X_{1}\right)\left(X_{2}-X_{3}\right) \dot{X}_{2}^{2}\right)\right\} \\
& +\left[a b-(b+\xi) \ell_{1}\right]\left[C_{1}-a_{11} X_{1}-a_{12} X_{2}\right. \\
& -a_{13} X_{3}+\left(b+X_{1}\right) \dot{X}_{2}^{2} \\
& \left.\left.+\left(A X_{1}+B X_{3}+a \dot{X}_{3}^{2}\right) X_{2}\right]\right\}, \\
& +\omega^{2}\left\{-X_{1} X_{2} \cos ^{2} \varphi_{0}-2 h \sin \omega t \cos \varphi_{0}\right. \\
& +\left[\left(b+X_{1}\right) X_{2} X_{3}+a\left(X_{3}^{2}-1\right)-b\right] \cos \varphi_{0} \sin \varphi_{0} \\
& +\frac{1}{b} f_{3}-c_{11} X_{1}-c_{12} X_{2}-c_{13} X_{3} \\
& +\frac{\ell_{1}}{a X_{3}}\left[C_{1}-a_{11} X_{1}-a_{12} X_{2}-a_{13} X_{3}+\left(b+X_{1}\right) \dot{X}_{2}^{2}\right. \\
& \left.+\left(A X_{1}+B X_{3}+a X_{3}^{2}\right) X_{2}\right]+\frac{1}{a H X_{3}} \\
& +\left(b+X_{1}\right)\left(f_{3}-c_{11} X_{1}-c_{12} X_{2}-c_{13} X_{3}\right) \\
& +b_{12} X_{2}+b_{13} X_{3}+g \sin \varphi_{0} \\
& \left.+b X_{3}\right)
\end{aligned}
$$

$$
\begin{aligned}
& \left.+X_{1} X_{2} \sin ^{2} \varphi_{0}\right\} \\
& \left.\left.+2 \dot{X}_{1} \dot{X}_{2}-\left(b+X_{1}\right)\left(X_{2}-X_{3}\right) \dot{X}_{2}^{2}\right)\right\} \\
& +\left[a b-\left(b+X_{1}\right) \ell_{1}\right] \\
& \cdot\left\{C_{1}-a_{11} \xi-a_{12} X_{2}-a_{13} X_{3}\right. \\
& \left.\left.\left.+\left(b+X_{1}\right) \dot{X}_{2}^{2}+\left(A X_{1}+B X_{3}+a \dot{X}_{3}^{2}\right) X_{2}\right\}\right\}\right\} \\
\dot{X}_{6} & =-\frac{1}{H}\left\{b\left(X_{2}-X_{3}\right)\right. \\
& \cdot\left[a_{11} X_{1}+a_{12} X_{2}+a_{13} X_{3}-\left(A X_{1}+B X_{3}\right) X_{2}\right] \\
& -b\left(b_{11} X_{1}+b_{12} X_{2}+b_{13} X_{3}\right) \\
& +\left(b+X_{1}\right)\left(c_{11} X_{1}+c_{12} X_{2}+c_{13} X_{3}\right) \\
& -b C_{1}\left(X_{2}-X_{3}\right) \\
+ & b \omega^{2} \cos \varphi_{0}\left(X_{1} X_{2} \cos \varphi_{0}+2 h \sin \omega t\right) \\
& -\left(b+X_{1}\right) f_{3}-b g \sin \varphi_{0} \\
+ & b \omega^{2}\left\{a+b-X_{3}\left[\left(b+X_{1}\right) X_{2}+a X_{3}\right]\right\} \\
& +\cos \varphi_{0} \sin \varphi_{0} \\
& -b\left[2 \dot{X}_{1} \dot{X}_{2}+X_{2}\right. \\
+ & \left.\left.\left(\omega^{2} X_{1} \sin ^{2} \varphi_{0}+a\left(X_{2}-X_{3}\right) \dot{X}_{3}^{2}\right)\right]\right\} .
\end{aligned}
$$

The following initial conditions are required to achieve the numerical solution of (18), by using the fourth-order RungeKutta method of ode45 solver in framework of Matlab program,

$$
\begin{aligned}
& X_{1}(0)=0.001, \\
& X_{2}(0)=0.1, \\
& X_{3}(0)=0.01, \\
& \dot{X}_{4}(0)=0, \\
& \dot{X}_{5}(0)=0, \\
& \dot{X}_{6}(0)=0
\end{aligned}
$$

in addition to the following physical parameters of the considered model:

$$
\begin{aligned}
& m=50 \mathrm{~kg}, \\
& g=9.8 \mathrm{~m} \cdot \mathrm{s}^{-2}, \\
& J_{1}=8 \mathrm{~kg} \cdot \mathrm{m}^{2}, \\
& \ell=0.7 \mathrm{~m}, \\
& \omega=4 \mathrm{rad} \cdot \mathrm{s}^{-1}, \\
& a=0.5 \mathrm{~m},
\end{aligned}
$$




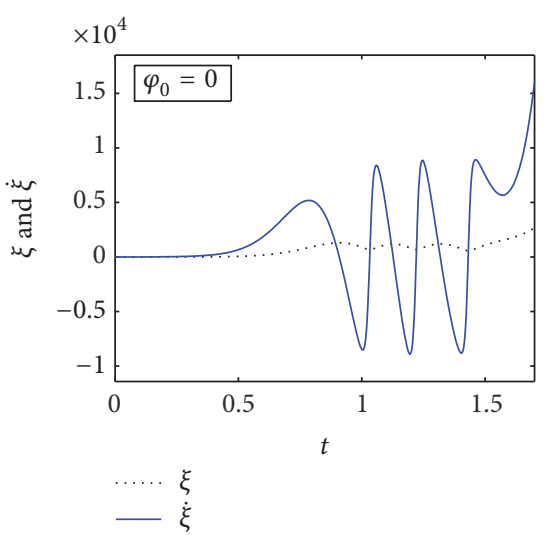

(a)

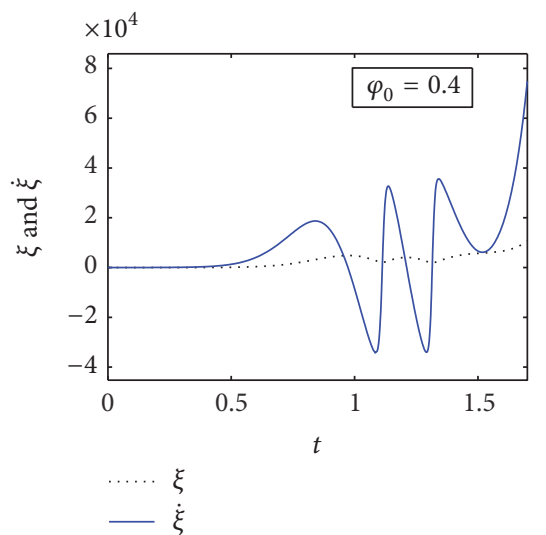

(d)

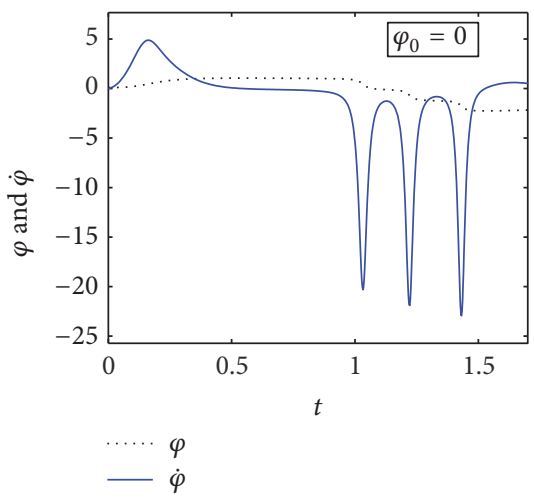

(b)

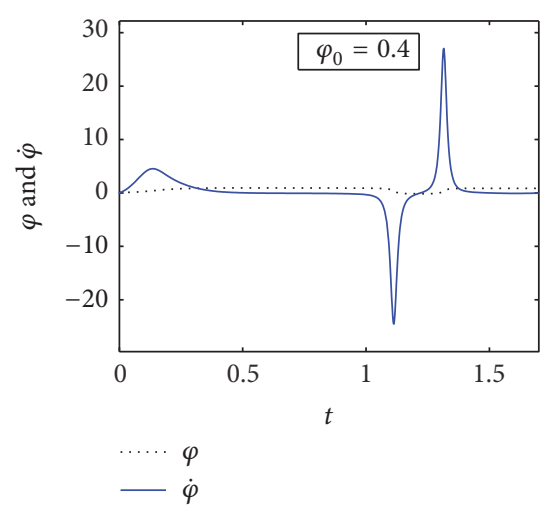

(e)

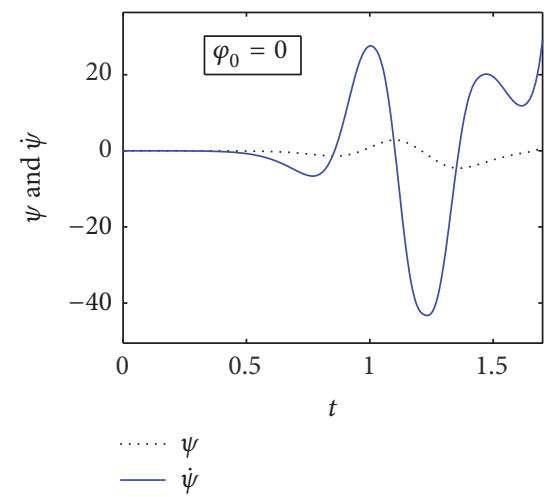

(c)

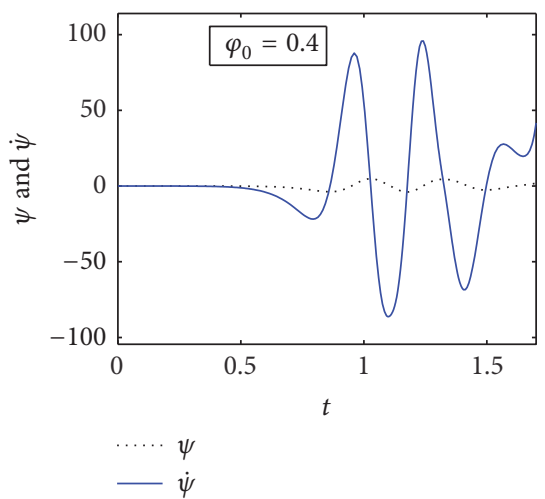

(f)

Figure 2: Variation of the solutions and their derivatives versus time $t$ when $b=3 \mathrm{~m}, h=4.5$, and $a=0.5 \mathrm{~m}$ : (a, d) show the effect of $t$ on the behavior of $\xi$ and $\dot{\xi}$ waves when $\varphi_{0}=0$ and $\varphi_{0}=0.4$ rad, respectively, (b, e) show the effect of $t$ on the behavior of $\varphi$ and $\dot{\varphi}$ waves when $\varphi_{0}=0$ and $\varphi_{0}=0.4 \mathrm{rad}$, respectively, and (c, f) show the effect of $t$ on the wave that describes the behavior of $\psi$ and $\dot{\psi}$ when $\varphi_{0}=0$ and $\varphi_{0}=0.4 \mathrm{rad}$, respectively.

$$
\begin{aligned}
b & =(0,3) \mathrm{m}, \\
\varphi_{0} & =(0,0.4) \mathrm{rad} \\
h & =(2.5,4.5), \\
t & =0 \longrightarrow 1.7 \mathrm{~min} .
\end{aligned}
$$

Figure 2 shows the variation of the solutions $\xi, \varphi, \psi$ and their derivatives $\dot{\xi}, \dot{\varphi}, \dot{\psi}$ against time $t$ when $\varphi_{0}=0$ and $\varphi_{0}=$ $0.4 \mathrm{rad}$. This figure is drawn at $b=3 \mathrm{~m}, h=2.4$, and $a=0.5 \mathrm{~m}$. The variations of $\xi, \varphi$, and $\psi$ with $\dot{\xi}, \dot{\varphi}$, and $\dot{\psi}$, respectively, are illustrated in Figure 3, namely, the phase plane diagrams that are represented in Figures 3(a), 3(b), $3(\mathrm{c})$ and $3(\mathrm{~d}), 3(\mathrm{e}), 3(\mathrm{f})$ when $\varphi_{0}=0$ and $\varphi_{0}=0.4 \mathrm{rad}$, respectively, with the same other parameters that are taken into consideration in Figure 2.

In these figures, our principle aim is to investigate the effect of increasing time on the motion of pendulum.

According to the calculations depicted in Figure 2(a), we found that when $\varphi_{0}=0$, the wave of the elongation $\xi$ grows up with the increasing of time till $t=0.9 \mathrm{~min}$. After that both of the elongation $\xi$ and its derivative $\dot{\xi}$ fluctuate between increasing and decreasing when time reaches $t=1.43 \mathrm{~min}$. Thus the wave of the solution $\xi$ is stable; see the phase plane Figure 3(a). With the passing of time, one can observe that $\xi$ and $\dot{\xi}$ are growing quickly, so the motion will be unstable after $t=1.43 \mathrm{~min}$. The rage behavior of both $\xi$ and $\dot{\xi}$ is due to the weight of the rigid body and the values of the principal moments of inertia. Consequently, we expect that behavior of elongation becomes greater as observed in Figures 2(a) and 2(d). Moreover, the variation of the spring between stretching and contraction is consistent with the phase plane diagrams represented in Figures 3(a) and 3(d).

It is worthwhile to notice from Figure 2(b) that when time $t$ increases from $t=0$ to $t=0.4 \mathrm{~min}$, the behavior of the angle $\varphi$ increases gradually to reach the value $\varphi \simeq 1 \mathrm{rad} \simeq 57^{\circ}$ and then decreases slowly to reach $\varphi \simeq 0.8 \mathrm{rad} \simeq 46^{\circ}$ during the time period $t \in] 0.4,0.9[\mathrm{~min}$. After $t=0.9 \mathrm{~min}$, the decline of the wave becomes quickly to reach $\varphi \simeq-2.3 \mathrm{rad} \simeq$ $-132^{\circ}$ at the end of time period (minus sign indicates opposite direction). This is not possible because $\varphi$ must belong to the interval ] $-\pi / 2, \pi / 2]$. So, the motion of the wave is unstable as it is manifest from Figure 3(b). On the other hand, $\dot{\varphi}$ increases till $t \simeq 0.2 \mathrm{~min}$ and then fluctuates as indicated from Figure 2(b). 


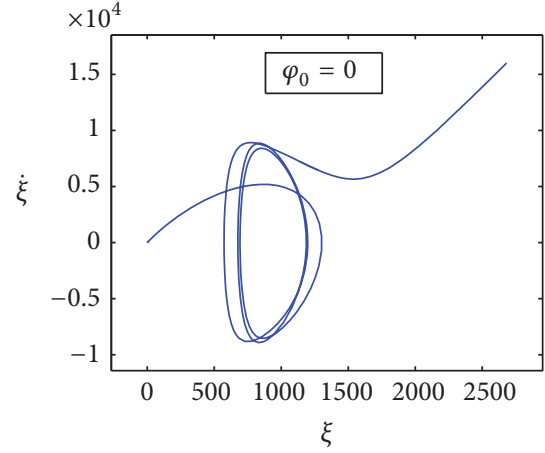

(a)

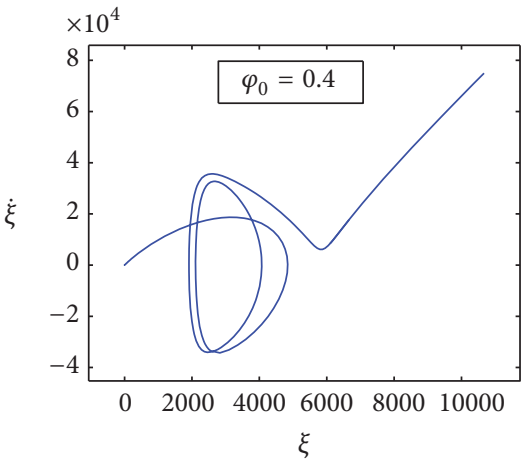

(d)

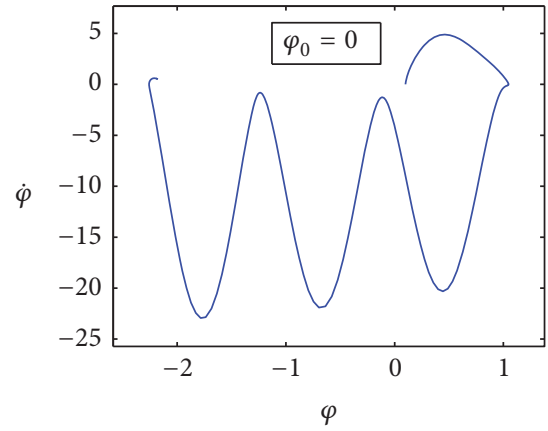

(b)

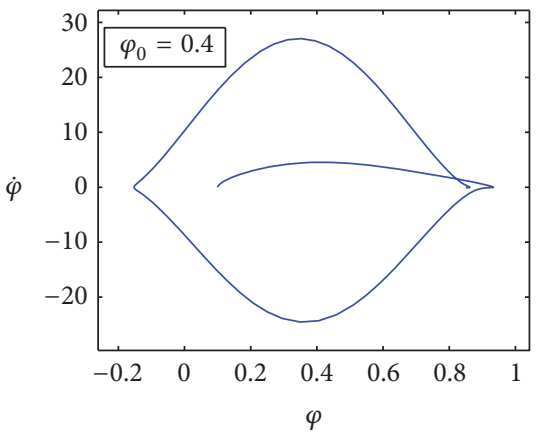

(e)

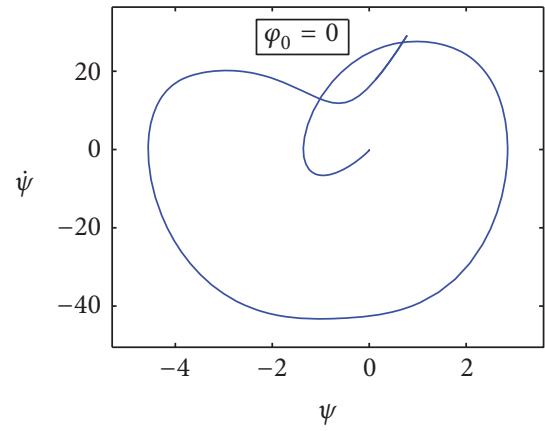

(c)

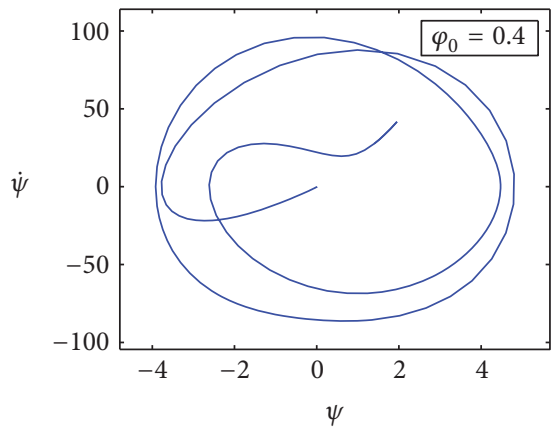

(f)

FIGURE 3: The phase plane diagram when $b=3 \mathrm{~m}, h=4.5$, and $a=0.5 \mathrm{~m}$ : (a, d) represent the variation of the amplitude $\xi$ with its velocity $\dot{\xi}$ at $\varphi_{0}=0$ and $\varphi_{0}=0.4 \mathrm{rad}$, respectively, (b, e) represent the variation of the amplitude $\varphi$ with its velocity $\dot{\varphi}$ at $\varphi_{0}=0$ and $\varphi_{0}=0.4 \mathrm{rad}$, respectively, and (c, f) represent the variation of the amplitude $\psi$ with its velocity $\dot{\psi}$ at $\varphi_{0}=0$ and $\varphi_{0}=0.4$ rad, respectively.

The graphs displayed in Figures 2(c) and 3(c) describe the variation of the ( $\psi$ and $\dot{\psi})$ against time and the phase plane diagram $\left(\dot{\psi}\right.$ with $\psi$ ), respectively, when $\varphi_{0}=0$. It is clear that when time belongs to the period $[0,0.43] \mathrm{min}$, the angle $\psi$ remains stationary, and then its wave oscillates between decreasing and increasing till $t=1.43 \mathrm{~min}$. After that time, the angle $\psi$ increases up to the end of time interval and consequently the motion will be stable as seen from Figure 3(c) during the period $0<t \leq 1.43$. It is obvious from Figure 2(c) that the behavior of $\dot{\psi}$ remains stationary to some extent through the time interval $[0,0.6] \mathrm{min}$ and then oscillates between increasing and decreasing till $t=1.7 \mathrm{~min}$.

It should be noticed that when $\varphi_{0}=0.4 \mathrm{rad}$, the stretching on the string $\xi$ increases gradually till the time $t$ becomes $0.9 \mathrm{~min}$, and then $\xi$ and $\dot{\xi}$ oscillate between increasing and decreasing when the time reaches the end of time interval; see Figure 2(d). Consequently, the wave of the solution is stable as seen from the phase plane Figure 3(d).

An inspection of the graphs depicted in Figure 2(e) shows that the wave describing the behavior of the angle $\varphi$ increases gradually from $\varphi=0$ at $t=0$ to its maximum value $\varphi \simeq$ $0.9 \mathrm{rad} \simeq 56^{\circ}$ at $t=0.4 \mathrm{~min}$ and then decreases slowly at $t \simeq 1 \mathrm{~min}$ to reach its minimum value $\varphi \simeq-0.19 \mathrm{rad} \simeq-11^{\circ}$ (minus sign indicates opposite direction) at $t \simeq 1.26 \mathrm{~min}$. With the increasing of time, the wave grows again to reach the value $\varphi \simeq 0.85 \mathrm{rad} \simeq 49^{\circ}$ at $t \simeq 1.39 \mathrm{~min}$. Thus, the motion is stable as it is manifest from Figure 3(e). On the other hand, $\dot{\varphi}$ increases and decreases as indicated from Figure 2(e).

Also, it is remarkable from Figure 2(f) that the behavior of the angle $\psi$ remains steady till $t=0.5 \mathrm{~min}$; then its wave oscillates between decreasing and increasing. Consequently the motion will be stable as seen from Figure 3(f). It is worthwhile to notice also from Figure 2(f) that the behavior of $\dot{\psi}$ oscillates between increasing and decreasing.

From the above observations, we can conclude that the motion of our model is more stable when $\varphi_{0}=0.4 \mathrm{rad}$ than when $\varphi_{0}=0$. This highlights the importance of the effect of $\varphi_{0}$ value on the motion. It is worthwhile to notice that the comparison between the solutions $\xi, \varphi$, and $\psi$ included in Figures 2(a), 2(b), and 2(c) with the corresponding Figures 2(d), 2(e), and 2(f) reveals that the amplitude of the waves decreases when $\varphi_{0}$ increases from 0 to $0.4 \mathrm{rad}$. On the other hand, the comparison between their derivatives shows that the amplitude of the waves increases when $\varphi_{0}$ increases. Figure 4 shows the variation of $(\xi, \dot{\xi}),(\varphi, \dot{\varphi})$, and $(\psi, \dot{\psi})$ with time $t$ when $\varphi_{0}$ changes from 0 for Figures $4(\mathrm{a}), 4(\mathrm{~b})$, and $4(\mathrm{c})$ to $0.4 \mathrm{rad}$ for Figures $4(\mathrm{~d}), 4(\mathrm{e})$, and $4(\mathrm{f})$ at the same values of other parameters $b=3 \mathrm{~m}, h=4.5$, and $a=0.5 \mathrm{~m}$. According to the calculations depicted in these figures, we can consider these figures as a rotation of the corresponding parts of Figure 3 with time to observe the bending and crossing of the resulting curves. 


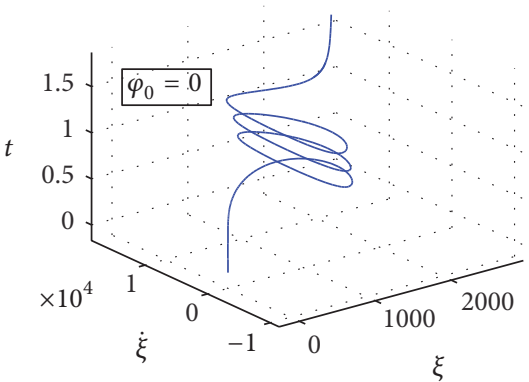

(a)

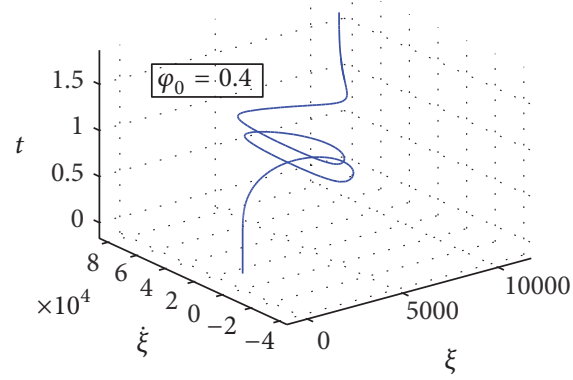

(d)

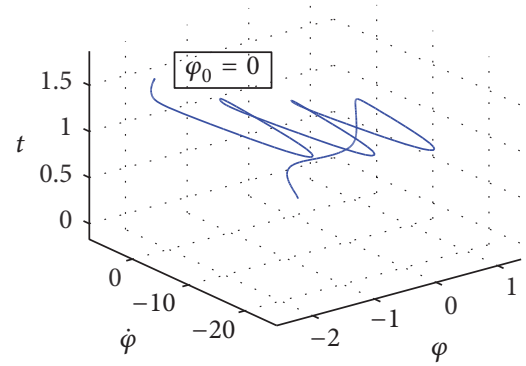

(b)

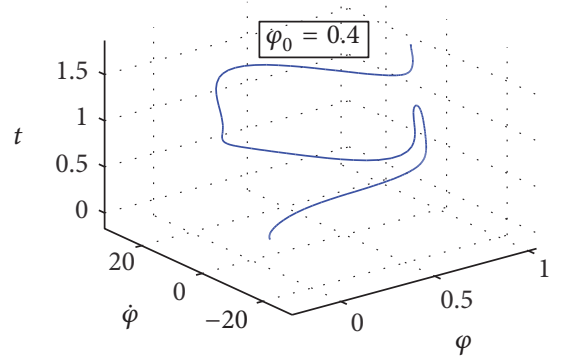

(e)

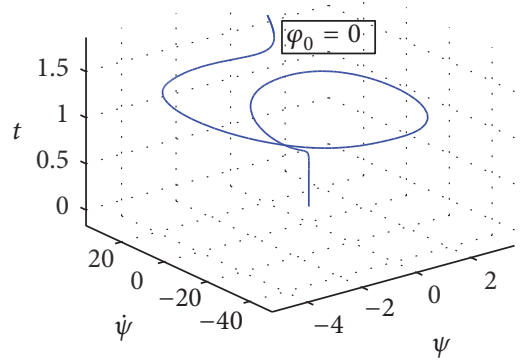

(c)

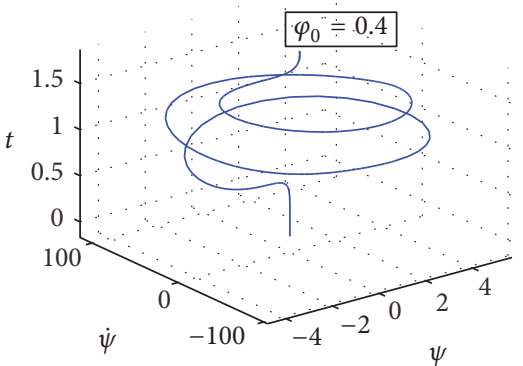

(f)

FiguRE 4: The 3D pattern when $b=3 \mathrm{~m}, h=4.5$, and $a=0.5 \mathrm{~m}:(\mathrm{a}, \mathrm{d})$ indicate the variation of $\xi$ and $\dot{\xi}$ versus $t$ when $\varphi_{0}=0$ and $\varphi_{0}=0.4 \mathrm{rad}$, respectively, (b, e) indicate the variation of $\varphi$ and $\dot{\varphi}$ versus $t$ when $\varphi_{0}=0$ and $\varphi_{0}=0.4$ rad, respectively, and (c, f) indicate the variation of $\psi$ and $\dot{\psi}$ versus $t$ when $\varphi_{0}=0$ and $\varphi_{0}=0.4 \mathrm{rad}$, respectively.

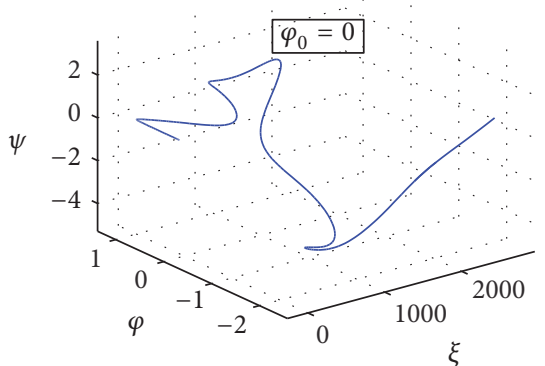

(a)

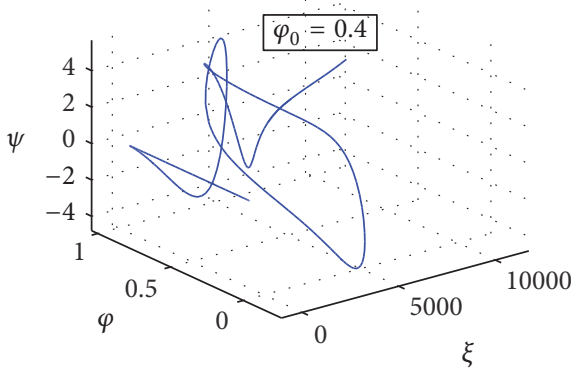

(c)

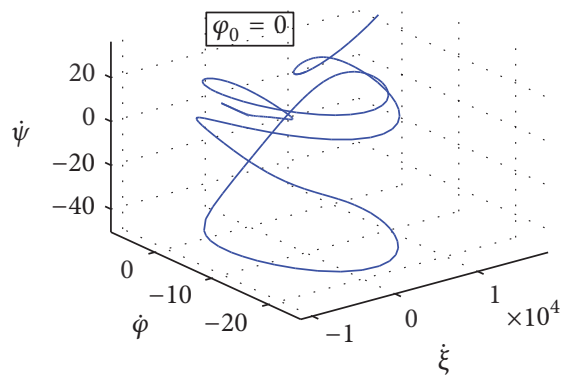

(b)

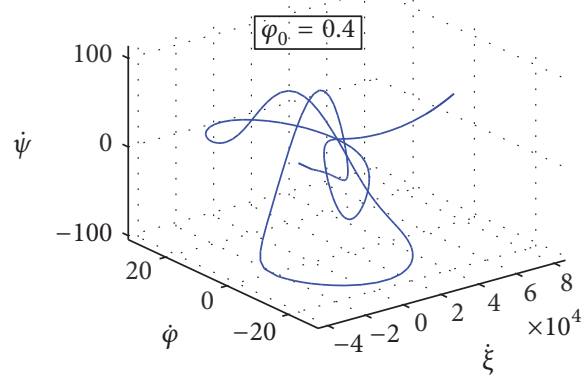

(d)

Figure 5: The 3D diagrams when $b=3 \mathrm{~m}, h=4.5$, and $a=0.5 \mathrm{~m}$ : (a, c) elucidate the variation of $\xi$ and $\varphi$ versus $\psi$ when $\varphi_{0}=0$ and $\varphi_{0}=0.4 \mathrm{rad}$, respectively, and (b, d) elucidate the variation of $\dot{\xi}$ and $\dot{\varphi}$ versus $\dot{\psi}$ when $\varphi_{0}=0$ and $\varphi_{0}=0.4 \mathrm{rad}$, respectively. 


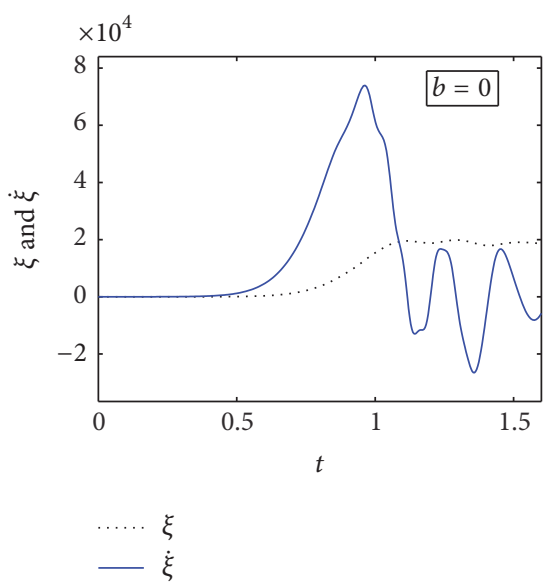

(a)

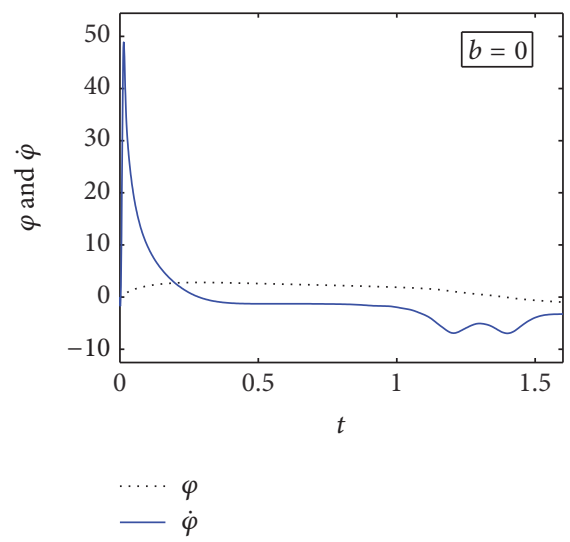

(b)

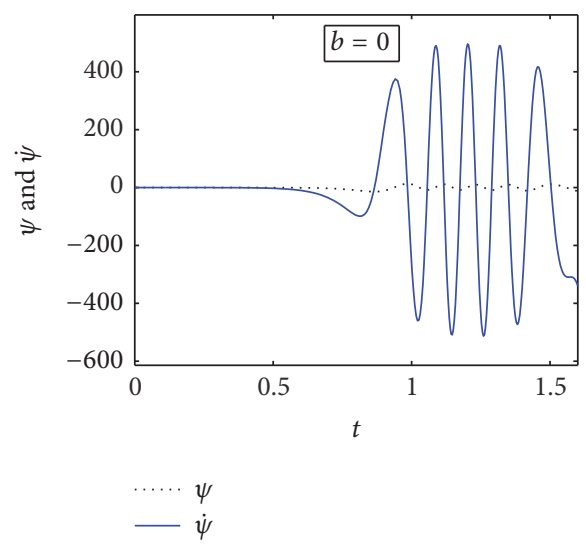

(c)

Figure 6: (a), (b), and (c) explain the variation of the solutions $\xi, \varphi$, and $\psi$ with their derivatives $\dot{\xi}, \dot{\varphi}$, and $\dot{\psi}$ via time $t$, respectively, when $b=0, h=4.5, a=0.5 \mathrm{~m}$, and $\varphi_{0}=0.4 \mathrm{rad}$.

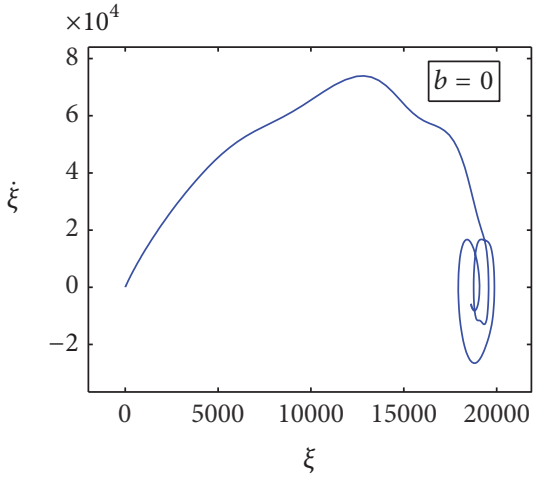

(a)

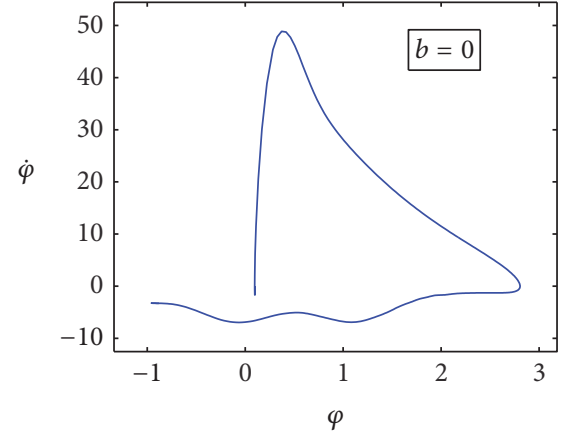

(b)

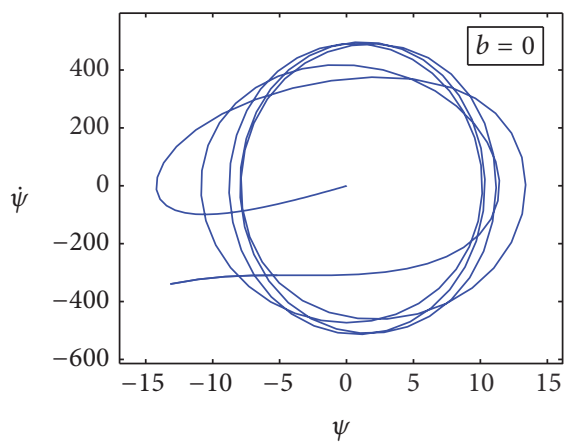

(c)

Figure 7: The phase plane diagrams between amplitudes and their velocities at $b=0, h=4.5, a=0.5 \mathrm{~m}$, and $\varphi_{0}=0.4 \mathrm{rad}$ : (a) shows the influence of $\xi$ on $\dot{\xi}$, (b) shows the effect of $\varphi$ on $\dot{\varphi}$, and (c) shows the variation of $\psi$ with $\dot{\psi}$.

Figures 5(a), 5(c) and 5(b), 5(d) represent 3D plots that illustrate the variation of the solutions $\xi, \varphi$ via $\psi$ and $\dot{\xi}, \dot{\varphi}$ via $\dot{\psi}$, respectively, for different values of $\varphi_{0}$ when $b=$ $3 \mathrm{~m}, h=4.5$, and $a=0.5 \mathrm{~m}$. The graphs displayed in parts of Figure 6 show the variation of $(\xi, \dot{\xi}),(\varphi, \dot{\varphi})$, and $(\psi, \dot{\psi})$ against time $t$ when $b=0$ with consideration of the parameters $\varphi_{0}=0.4 \mathrm{rad}, h=4.5$, and $a=0.5 \mathrm{~m}$. The corresponding phase plane between the amplitudes $\xi, \varphi, \psi$ and their derivatives $\dot{\xi}, \dot{\varphi}, \dot{\psi}$ is represented in parts of Figure 7 . Inspection of the graph depicted in Figure 6(a) shows that when time $t$ increases from 0 to $0.45 \mathrm{~min}$ the behavior of the solution $\xi$ remains stationary and quickly growing during the time interval $t \in] 0.45,1.05$ [ min and then oscillates till the end of time interval. This indicates that the motion is stable as seen from Figure 7(a). On the other side, the behavior of the derivative $\dot{\xi}$ remains approximately stationary during the interval $t \in[0,0.45] \mathrm{min}$ and then fluctuates with the increasing of time; see Figure 6(a).
By the same way, we can observe that the wave of the angle $\varphi$ increases through a short time to reach its maximum value $\varphi \simeq 2.7 \mathrm{rad} \simeq 155^{\circ}$ at $t \simeq 0.23 \mathrm{~min}$, taking into consideration that $-\pi / 2<\varphi<\pi / 2$, and then decreases slowly to reach its minimum value $\varphi \simeq-0.9 \mathrm{rad} \simeq-52^{\circ}$ at the end of time interval; see Figure 6(b). This indicates that the motion is close to be stable as observed from the phase plane Figure 8(b). As seen from Figure 6(b), $\dot{\varphi}$ increases and decreases quickly during the period $t \in$ $[0,0.1]$ min to reach its minimum value at the end of time interval.

The variation of $\psi$ and $\dot{\psi}$ with time is illustrated in Figure 6(c). In this figure our main goal is to examine the influence of time on the motion of pendulum. It is clear that the behavior of $\psi$ and $\dot{\psi}$ remains stationary (to some extent) when $t \in[0,0.5] \mathrm{min}$; then their waves fluctuate till the end of time interval. Consequently the motion is stable as seen from the phase plane diagram, Figure $7(\mathrm{c})$. The comparison between parts of Figure 6 with the corresponding Figures 


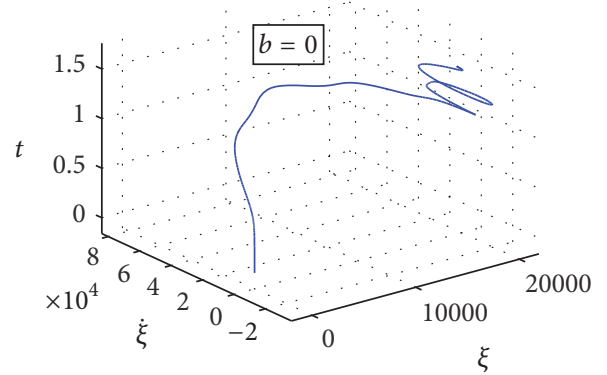

(a)

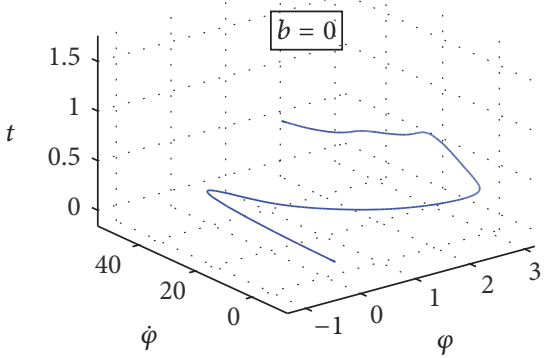

(b)

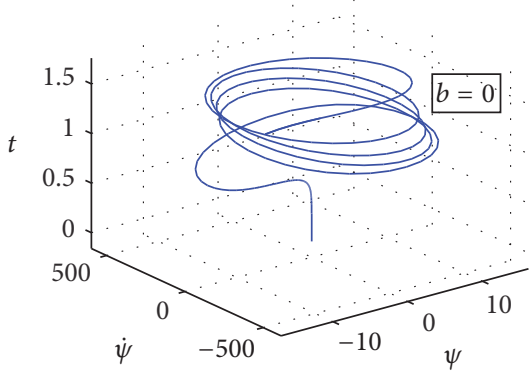

(c)

Figure 8: The 3D plots at $b=0, h=4.5, a=0.5 \mathrm{~m}$, and $\varphi_{0}=0.4 \mathrm{rad}$ : (a) illustrates the variation of $\xi$ and $\dot{\xi}$ via $t$, (b) illustrates the variation of $\varphi$ and $\dot{\varphi}$ via $t$, and (c) illustrates the variation of $\psi$ and $\dot{\psi}$ via $t$.

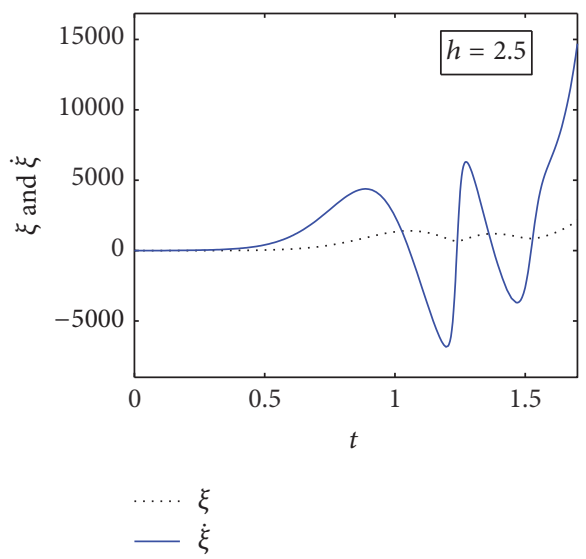

(a)

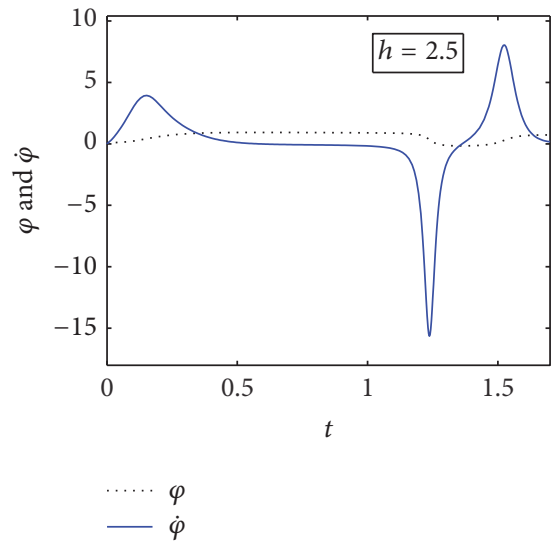

(b)

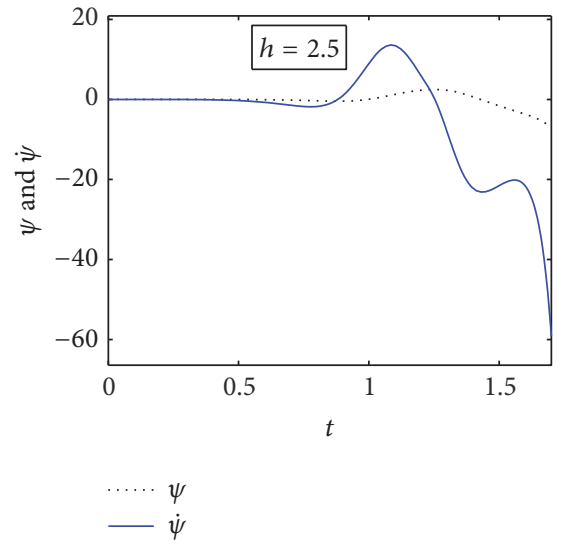

(c)

Figure 9: (a), (b), and (c) demonstrate the variation of ( $\xi$ and $\dot{\xi}$ ), ( $\varphi$ and $\dot{\varphi})$, and ( $\psi$ and $\dot{\psi}$ ) against time $t$, respectively, at $b=3 \mathrm{~m}, h=2.5$, $a=0.5 \mathrm{~m}$, and $\varphi_{0}=0.4 \mathrm{rad}$.

2(d), 2(e), and 2(f) shows that when $b$ changes from 0 to $3 \mathrm{~m}$ the amplitude of the waves decreases. Also, the motion will be more stable when $b=3 \mathrm{~m}$ than when $b=0$ as seen from the corresponding phase plane diagrams, that is, Figures $3(d)$, $3(\mathrm{e}), 3(\mathrm{f})$ and $7(\mathrm{a}), 7(\mathrm{~b}), 7(\mathrm{c})$, respectively.

On the other hand, parts of Figure 8 show 3D plots that describe the variation of the solutions and their derivative via time when $b=0, h=4.5, \varphi_{0}=0.4 \mathrm{rad}$, and $a=0.5 \mathrm{~m}$. The plots displayed in the mentioned parts show bending and crossing of the resulting curves.

Figures 9(a), 9(b), and 9(c) show the variation of the solutions $\xi, \varphi, \psi$ and their derivatives $\dot{\xi}, \dot{\varphi}, \dot{\psi}$ with time $t$ when $h=2.5$ for the given values of other parameters $b=3 \mathrm{~m}$, $\varphi_{0}=0.4 \mathrm{rad}$, and $a=0.5 \mathrm{~m}$. In view of the first part, we can conclude that when time $t$ increases, each of the waves $\xi$ and $\dot{\xi}$ oscillates between increasing and decreasing till $t=1.46 \mathrm{~min}$ and then increases gradually. So, the motion is stable as seen from Figure 10(a).

From a closer look on the second part of Figure 9(b) we can write, with the increasing of time, the behavior of $\varphi$ wave increases to reach its maximum value $\varphi \simeq 0.9 \mathrm{rad} \simeq 52^{\circ}$ at $t=0.43 \mathrm{~min}$ and then decreases slowly through the period $t \epsilon$ ]0.43, 1.19] min. After that, its behavior has a sharp decline in a few seconds (about $2.4 \mathrm{~s}$ ) and then increases till the end of time period and consequently the motion is stable; see Figure 10(b).

According to the calculations depicted in Figure 9(c), we can observe that the waves describing $\psi$ and $\dot{\psi}$ decrease slowly till $t=0.9 \mathrm{~min}$ and then increase and decline sharp. The phase plane Figure $10(\mathrm{c})$ shows that the behavior of $\psi$ is not stable.

When parts of Figure 9 and their phase plane parts (of Figure 10) are generally compared with the corresponding Figures 2(d), 2(e), and 2(f) and their phase plane Figures 3(d), $3(\mathrm{e})$, and 3(f), we can observe that amplitude of the wave increases when $h=4.5$ compared to when $h=2.5$ and the motion is more stable when $h=4.5$. An inspection of parts of Figure 11 reveals the $3 \mathrm{D}$ plots when $h=2.5$ with the same other data considered in Figures 9 and 10. Figure 10 shows the variation of the solutions $\xi, \varphi, \psi$ and their derivatives $\dot{\xi}, \dot{\varphi}, \dot{\psi}$ 


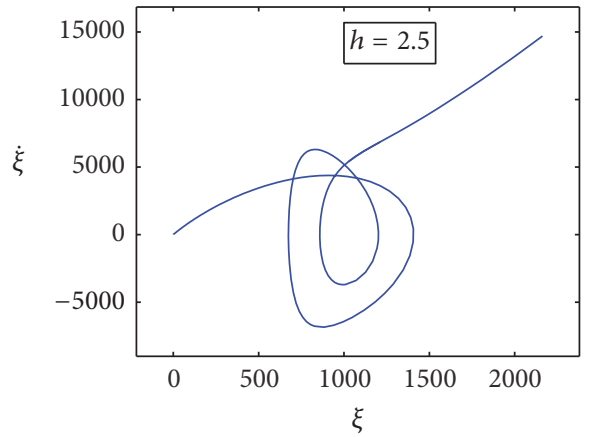

(a)

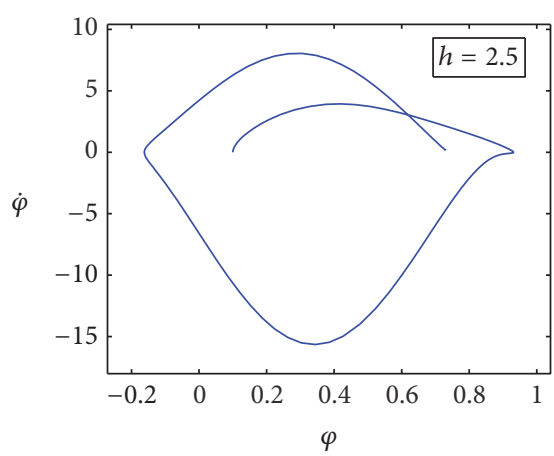

(b)

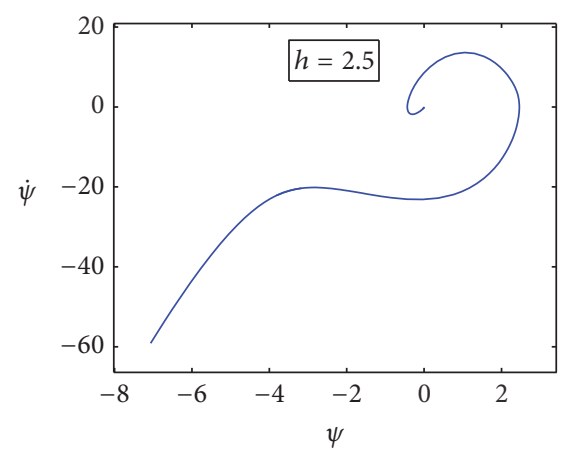

(c)

Figure 10: The phase plane diagrams which portray the relation between amplitudes and their velocities at $b=3 \mathrm{~m}, h=2.5, a=0.5 \mathrm{~m}$, and $\varphi_{0}=0.4 \mathrm{rad}$ : (a) describes the influence of $\xi$ on $\dot{\xi}$, (b) shows the effect of $\varphi$ on $\dot{\varphi}$, and (c) illustrates the variation of $\psi$ with $\dot{\psi}$.

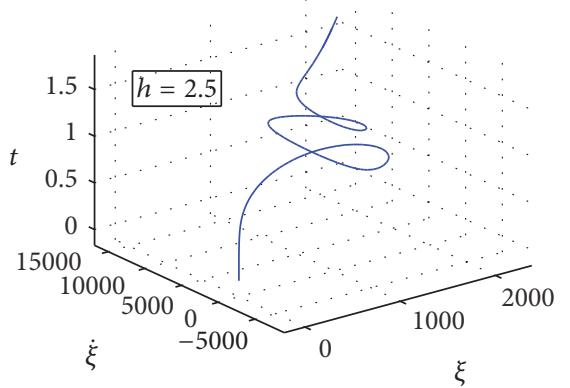

(a)

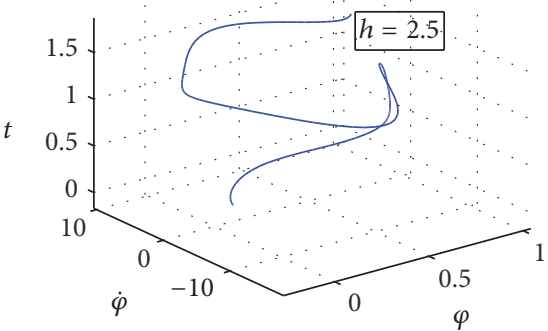

(b)

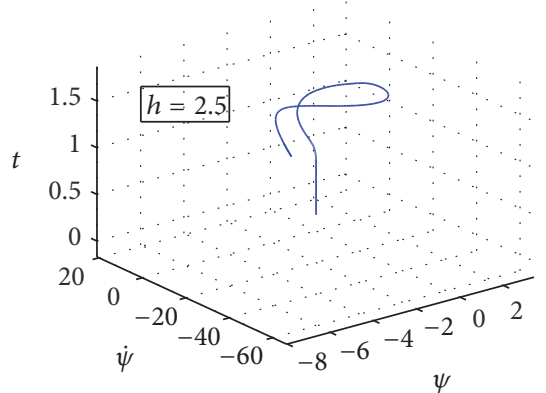

(c)

Figure 11: The 3D patterns at $b=3 \mathrm{~m}, h=2.5, a=0.5 \mathrm{~m}$, and $\varphi_{0}=0.4 \mathrm{rad}$ : (a) illustrates the variation of $\xi$ and $\dot{\xi}$ versus $t$, (b) illustrates the variation of $\varphi$ and $\dot{\varphi}$ versus $t$, (c) illustrates the variation of $\psi$ and $\dot{\psi}$ versus $t$.

with time $t$. It is worthwhile to notice that the comparison between Figures 4(d), 4(e), and 4(f) and Figures 11(a), 11(b), and 11(c) shows more bending and crossing of the curves in Figures 4(d), 4(e), and 4(f) when $h=4.5$ than the corresponding ones of Figure 11.

Now, we study the last case when $h=0$ with the same other data $b=3 \mathrm{~m}, \varphi_{0}=0.4 \mathrm{rad}$, and $a=0.5 \mathrm{~m}$. The obtained results are represented graphically in Figures 12(a), 12(b), and 12(c), while their phase plane diagrams are given in Figures 12(d), 12(e), and 12(f). At the first glance, we can conclude that this case is not stable, so it is very important to notice that the dimensionless parameter $h$ must take any value different from zero as it is pointed in Figure $2(h=4.5)$ and Figure $9(h=$ 2.5). This elucidates the importance of $h$ parameter on the motion.

\section{Conclusion}

A conclusion that may be made here is that the problem of the relative motion of a rigid body as a pendulum model is investigated. The governing deferential equations are obtained using Lagrange's equations. Mathematica package was utilized, in order to overcome the difficulties that appear in the separation of the second derivatives of the generalized coordinates $\xi, \varphi$, and $\psi$ for the nonlinear system (10). Computer codes are used to obtain the numerical solutions for system (14). These solutions are represented graphically using Matlab program, to study the influence of the different parameters on the motion. The good effect of the parameters $h, b$, and $\varphi_{0}$ on the motion is obvious from the mentioned plots. The motion of our model is more stable when the parameters $h, b$, and $\varphi_{0}$ take values run away from zero. This highlights the importance of the effect of these parameters on the motion. Such results have been confirmed by many works, such as Ismail [13] and Amer and Bek [14].

\section{Competing Interests}

The author declares that they have no competing interests. 


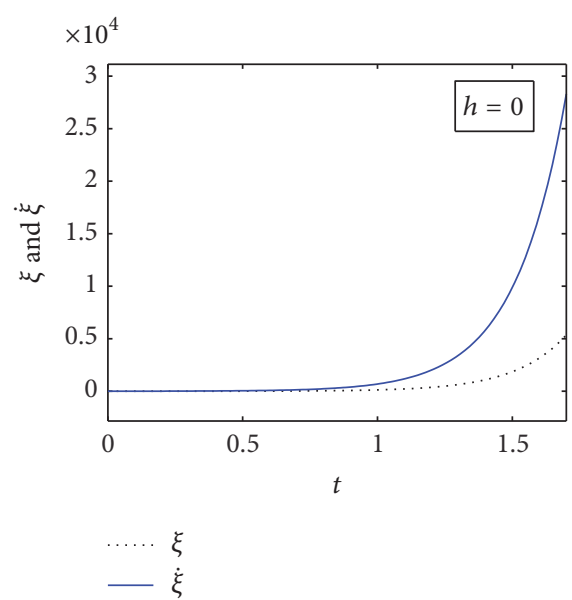

(a)

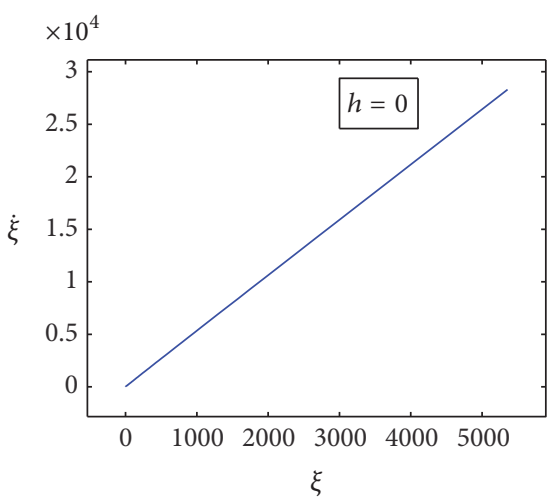

(d)
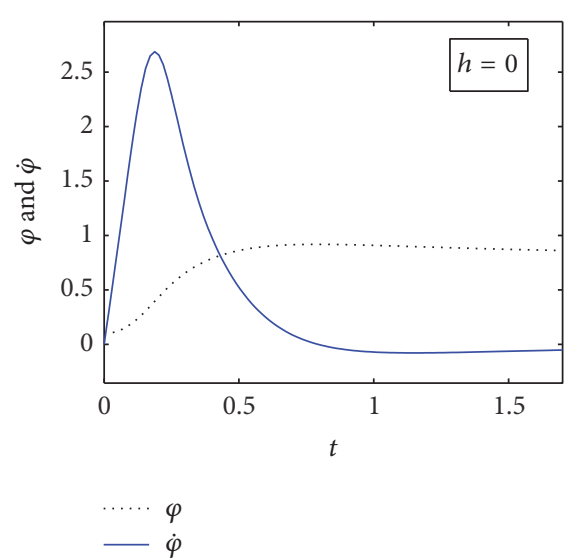

(b)

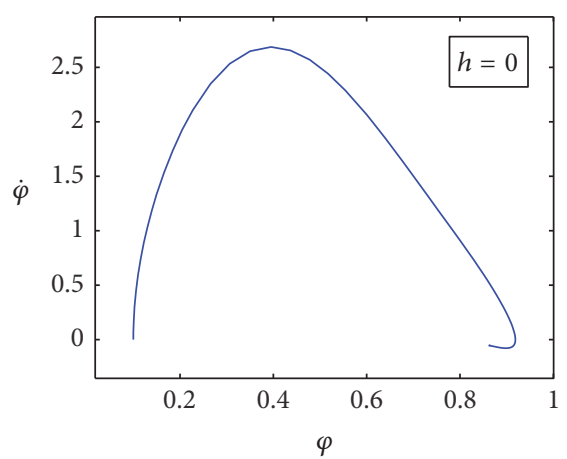

(e)

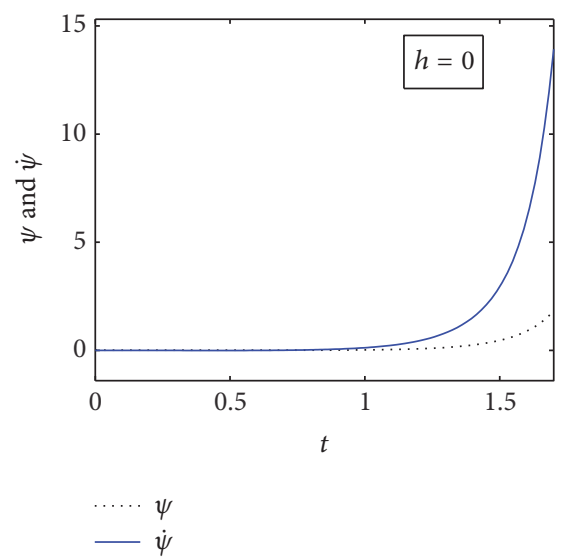

(c)

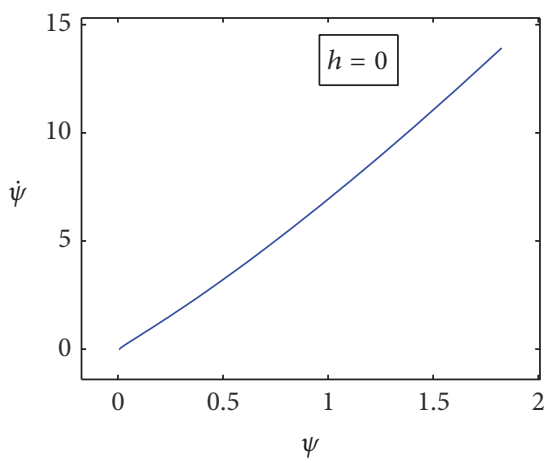

(f)

FIGURE 12: (a, b, and c) explain the variation of the solutions $\xi, \varphi$, and $\psi$ with their derivatives $\dot{\xi}, \dot{\varphi}$, and $\dot{\psi}$ via time $t$, respectively, when $b=3 \mathrm{~m}$, $h=0, a=0.5 \mathrm{~m}$, and $\varphi_{0}=0.4 \mathrm{rad}$; (d, e, and f) illustrate the variation of the solutions against their first derivatives for the same values of the considered parameters.

\section{References}

[1] P. Lynch, "Resonant motions of the three-dimensional elastic pendulum," International Journal of Non-Linear Mechanics, vol. 37, no. 2, pp. 345-367, 2002.

[2] A. A. Klimenko, Y. V. Mikhlin, and J. Awrejcewicz, "Nonlinear normal modes in pendulum systems," Nonlinear Dynamics, vol. 70, no. 1, pp. 797-813, 2012.

[3] S. Mori, H. Nishihara, and K. Furuta, "Control of unstable mechanical system control of pendulum," International Journal of Control, vol. 23, no. 5, pp. 673-692, 1976.

[4] C. C. Chung and J. Hauser, "Nonlinear control of a swinging pendulum," Automatica. A Journal of IFAC, vol. 31, no. 6, pp. 851-862, 1995.

[5] A. Shiriaev, A. Pogromsky, H. Ludvigsen, and O. Egeland, "On global properties of passivity-based control of an inverted pendulum," International Journal of Robust and Nonlinear Control, vol. 10, no. 4, pp. 283-300, 2000.

[6] A. S. Shiriaev, H. Ludvigsen, and O. Egeland, "Swinging up the spherical pendulum via stabilization of its first integrals," Automatica. A Journal of IFAC, the International Federation of Automatic Control, vol. 40, no. 1, pp. 73-85, 2004.

[7] M. N. Brearley, "The Simple Pendulum with Uniformly Changing String Length," Proceedings of the Edinburgh Mathematical Society, vol. 15, no. 1, pp. 61-66, 1966.
[8] S. J. Liao, "Second-order approximate analytical solution of a simple pendulum by the process analysis method," Journal of Applied Mechanics, Transactions ASME, vol. 59, no. 4, pp. $970-$ 975, 1992.

[9] W. K. Tso and K. G. Asmis, "Parametric excitation of a pendulum with bilinear hysteresis," Journal of Applied Mechanics, Transactions ASME, vol. 37, no. 4, pp. 1061-1068, 1970.

[10] A. H. Nayfeh, Perturbations Methods, Wiley-VCH, Weinheim, Germany, 2004.

[11] F. A. El-Barki, A. I. Ismail, M. O. Shaker, and T. S. Amer, "On the motion of the pendulum on an ellipse," Zeitschrift fur Angewandte Mathematik und Mechanik, vol. 79, no. 1, pp. 65-72, 1999.

[12] N. V. Stoianov, "On the relative periodic motions of a pendulum," Journal of Applied Mathematics and Mechanics, vol. 28, pp. 188-193, 1964.

[13] A. I. Ismail, "Relative periodic motion of a rigid body pendulum on an ellipse," Journal of Aerospace Engineering, vol. 22, no. 1, pp. 67-77, 2009.

[14] T. S. Amer and M. A. Bek, "Chaotic responses of a harmonically excited spring pendulum moving in circular path," Nonlinear Analysis. Real World Applications. An International Multidisciplinary Journal, vol. 10, pp. 3196-3202, 2009. 
[15] L. D. Akulenko, "Parametric control of oscillations and rotations of a compound pendulum (a swing)," Journal of Applied Mathematics and Mechanics, vol. 57, no. 2, pp. 301-310, 1993.

[16] M. A. Pinsky and A. A. Zevin, "Oscillations of a pendulum with a periodically varying length and a model of swing," International Journal of Non-Linear Mechanics, vol. 34, no. 1, pp. 105-109, 1999.

[17] M. Kamel, M. Eissa, and A. T. El-Sayed, "Vibration reduction of a nonlinear spring pendulum under multiparametric excitations via a longitudinal absorber," Physica Scripta, vol. 80, no. 2, Article ID 025005, 2009.

[18] M. Eissa, M. Kamel, and A. T. El-Sayed, "Vibration reduction of multi-parametric excited spring pendulum via a transversally tuned absorber," Nonlinear Dynamics, vol. 61, no. 1-2, pp. 109121, 2010.

[19] R. Starosta, G. Sypniewska-Kaminska, and J. Awrejcewicz, "Asymptotic analysis of kinematically excited dynamical systems near resonances," Nonlinear Dynamics. An International Journal of Nonlinear Dynamics and Chaos in Engineering Systems, vol. 68, no. 4, pp. 459-469, 2012.

[20] H. Moore, Matlab for Engineers, Pearson, 3rd edition, 2012.

[21] M. D. Ardema, Analytical Dynamics, Theory and Applications, Springer, Berlin, Germany, 2009.

[22] A. Tewari, Modern Control Design with Matlab and Similink, John Wiley and Sons Ltd, New York, NY, USA, 2002. 


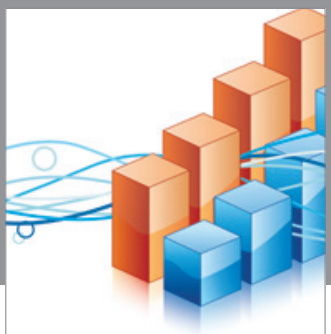

Advances in

Operations Research

vatem alat4

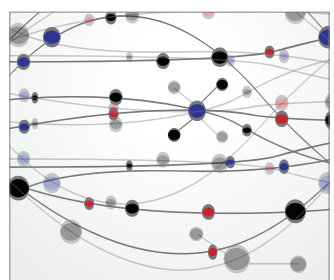

\section{The Scientific} World Journal
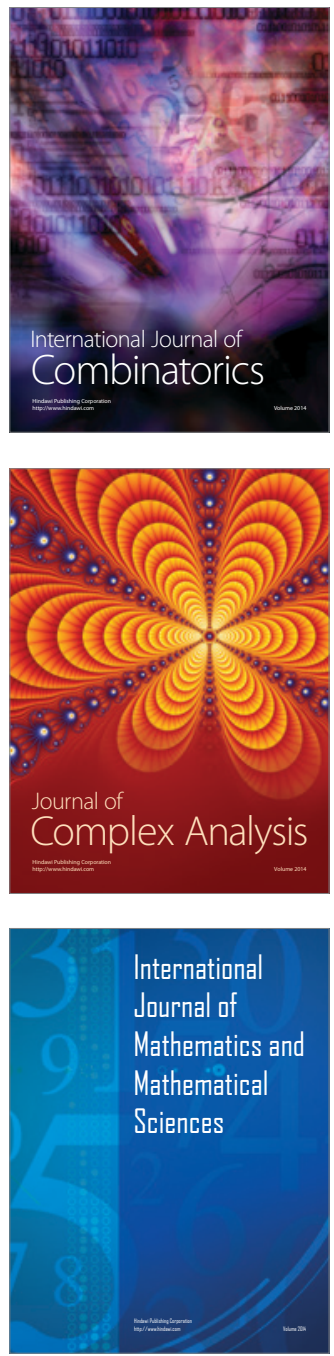
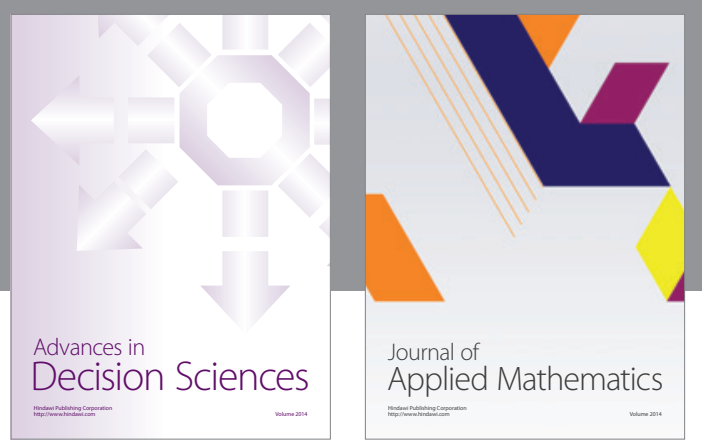

Algebra

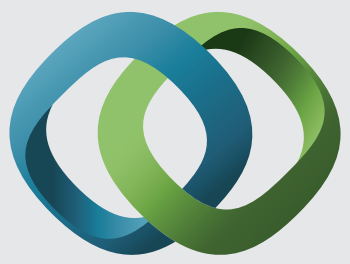

\section{Hindawi}

Submit your manuscripts at

https://www.hindawi.com
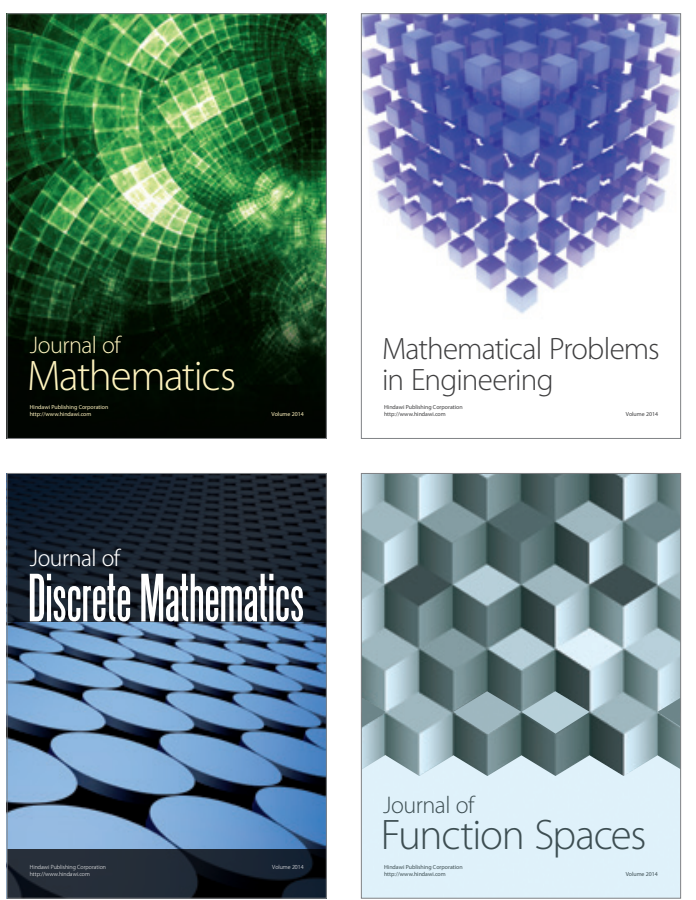

Mathematical Problems in Engineering
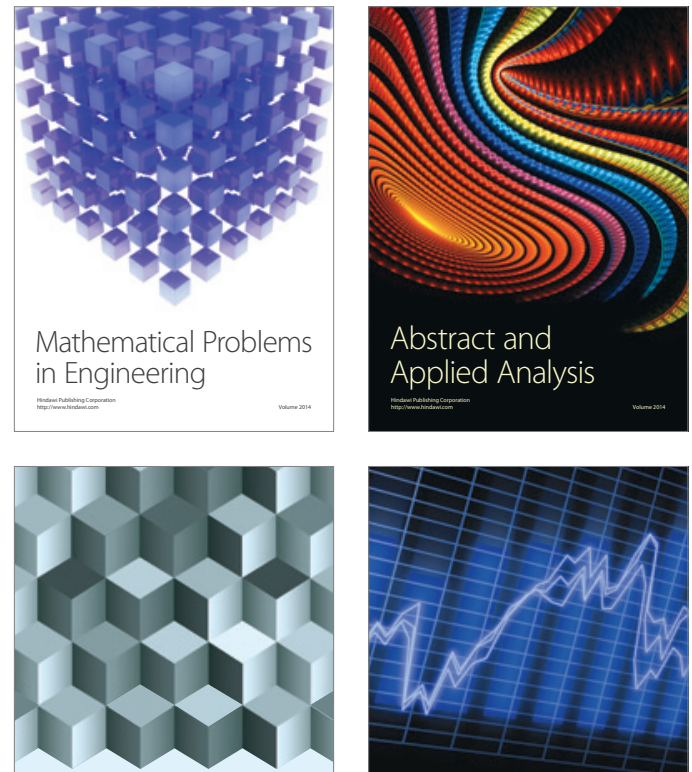

Journal of

Function Spaces

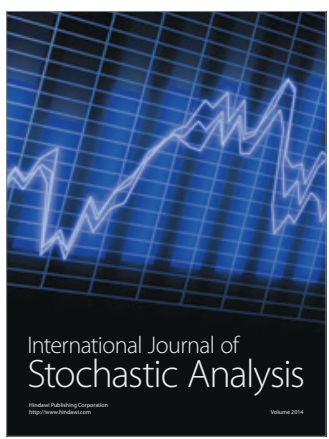

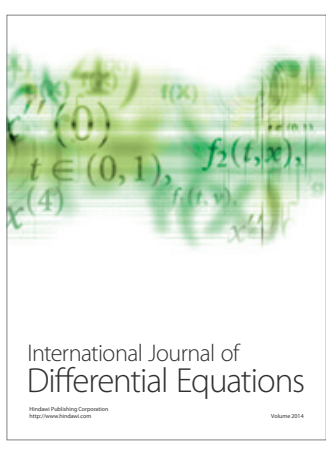
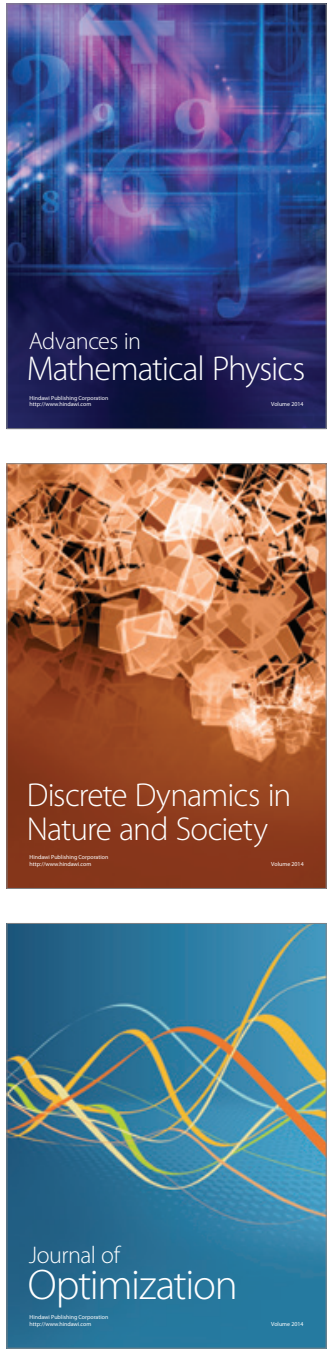